\title{
Bilateral Gonadblastoma and Dysgerminoma in an 18-Year-0ld Female Patient with 46xy, Sty Gene Mutation
}

\author{
Schmeink $\mathrm{CE}^{1 *}$, Van Altena $\mathrm{AM}^{1}$, Kasius $\mathrm{JC}^{1}$, Claahsen van der Grinten $\mathrm{HL}^{2}$ and Beerendonk $\mathrm{CCM}^{1}$ \\ ${ }^{1}$ Department of Obstetrics \& Gynecology, Netherlands \\ ${ }^{2}$ Department of Pediatric Endocrinologist, Netherlands \\ *Corresponding author: CE Schmeink, Department of Obstetrics \& Gynecology, Geert Grooteplein zuid 10,6525 GA, Nijmegen, Netherlands
}

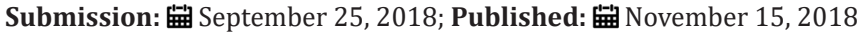

\begin{abstract}
Background: The need for prophylactic gonadectomy in XY gonadal dysgenesis patients is well known because of the risk of malignancy. However the timing of surgery is not prescribed and in case of an unexpected finding like a dysgerminoma the need for additional treatment is controversial.

Case: A 18-year-old adolescent with a 46XY disorder of sex development (DSD) and a SRY gene mutation underwent a laparoscopic gonadectomy. Pathology showed bilateral gonadoblastoma and dysgerminoma. Additional imaging showed no metastasis. After multidisciplinary consultation, expectant management was advised. In the first years of follow-up, there was no recurrence of disease.

Conclusion: This case report of a patient with bilateral gonadoblastoma and dysgerminoma contributes to the limited data available about this type of germ cell tumor and the required treatment and follow-up. A conservative approach was chosen, as completion surgery and adjuvant chemotherapy are controversial and recurrence of disease can be well treated with chemotherapy.
\end{abstract}

\section{Introduction}

The primordial gonad is bipotential and can differentiate into a testis or an ovary. Gonadal dysgenesis can be defined as an incomplete or defective formation of the gonads, as a result of a disturbed process of migration of the germ cells and/or their correct organization in the fetal gonadal ridge [1]. The SRY protein induces expression of the transcription factor SOX9. This results in formation of Sertoli cells which affect the functionality of fetal Leydig cells. These cells are the most important source of fetal testosterone production [2-4]. Therefore, mutations in the SRY gene can cause gonadal dysgenesis. Due to a lack of fetal testosterone and anti-Mullerian hormone (AMH) production, complete $\mathrm{XY}$ gonadal dysgenesis is associated with female external genital organs, intact Mullerian ducts and streak gonads $[4,5]$. These patients are usually not diagnosed at birth because of their normal female appearance, but they attend the clinic later with the absence of sex characteristics at pubertal age and primary amenorrhea. These girls have an increased risk of developing germ cell cancer [2,5].

Germ cell cancer arises from primordial germ cells or gonocytes and can be subdivided into two groups; seminomas/ dysgerminomas and non-seminomas, with carcinoma in situ (CIS) or gonadoblastoma (GB) as precursor lesion. GB and CIS are highly similar on mRNA expression level and immunohistochemical markers. The fact that digenetic gonads predominately contain dysgerminoma instead of various non-seminomas is likely related to the intra-abdominal localization of the gonad, and not due to a pathological difference between GB and CIS [1,2]. Gonadoblastoma is a pre-malignant neoplasm with a germ cell component liable to malignant transformation into invasive dysgerminoma [6,7] In the general population, gonadoblastoma and dysgerminoma are rare conditions with a prevalence of $0.2 \%$ and $1.6 \%$, respectively. (7) Patients with gonadal dysgenesis have an increased risk of developing a gonadoblastoma (up to 30\%) with a 50-60\% risk of malignant transformation of these gonadoblastoma to dysgerminoma. Therefore, prophylactic bilateral gonadectomy is recommended in patients diagnosed with gonadal dysgenesis $[2,4$, $5,8]$

Bilateralism is rare in malignant ovarian germ cell tumors with a reported prevalence of $4.3-6.9 \%$, rising to $10-15 \%$ in dysgerminoma and mixed tumors with dysgerminoma [9-12]. Furthermore, the combination of gonadoblastoma and dysgerminoma is also rare. A large review on SRY mutation carriers of whom $94 \%$ had a female phenotype looked at histology. It showed that in only 2, of the 61 cases in which gonadal histology was analyzed, a combination of gonadoblastoma and dysgerminoma was seen (3\%). Eighteen had gonadoblastoma (30\%), and there was 1 dysgerminoma (1\%) [2]. These data underline the rarity of our case of a18-year old female patient with 46XY, SRY gene mutation with a bilateral gonadoblastoma and dysgerminoma. 
With this case report we aim to increase general knowledge about the malignant potential of the gonads of patients with gonadal dysgenesis in $46 \mathrm{XY}$ conditions and the importance of early prophylactic bilateral gonadectomy. Furthermore, we want to discuss the management in case of malignancy and increase awareness for the lack of evidence for surgical staging and adjuvant chemotherapy.

\section{Case Presentation}

Our case is an 18-year-old patient, who was primarily seen by a pediatrician in a non-university hospital because of primary amenorrhea and signs of hyperandrogenism. Hormonal examination, karyotyping and DNA testing were performed: Follicle-stimulating hormone (FSH) 60IU/L (Ref: follicular 3.012.0; ovulation 4.0-25.0; luteal 1.5-9.0, post-menopause 15.0150.0; male 1.0-19.0U/l) , Luteinizing hormone (LH) 34IU/L (Ref: follicular 2.0-11.0; ovulation 15.0-100.0; luteal 1.0-13.0, postmenopause 10.0-60.0; male $<70 \mathrm{y} 1.0-9.0 \mathrm{U} / \mathrm{l}$ ) and testosterone $2.6 \mathrm{nmol} / \mathrm{l}$ (Ref: $0.5-2.4 \mathrm{nmol} / \mathrm{l}$ ) were elevated. The karyotype was found to be 46XY and a de novo SRY gene mutation was detected. The uterus appeared normal on ultrasound and MRI. On both sides of the uterus a structure was detected which appeared as gonads. After referral to a pediatric endocrinologist at the Radboud university medical Centre, additional blood tests were performed. Testosterone was again elevated 3.4nmol/l (Ref:0.5-2.4nmol/l) and lactatedehydrogenasis (LDH) 258U/l $(\operatorname{Re}<250)$ was slightly elevated. Other biochemical markers were normal, dehydro-epiandrosteron (DHEA) $17.4 \mathrm{nmol} / \mathrm{l}$ (ref 15-45), dihydrotestosterone $0.21 \mathrm{nnmol} / \mathrm{l}$ (ref $0.14-0.76$ ), progesterone $0.14 \mathrm{nnmol} / \mathrm{l}, 17-\mathrm{OH}-$ progesteron $1.77 \mathrm{nmol} / \mathrm{l}$, AMH 2.1Uug/l, alpha-fetoprotein $2.7 \mathrm{ug} / \mathrm{l}$ (ref < 10), $\beta$ HCG<1.0ng/l (ref<2), Inhibin B 10ng/l (ref 10-200).

With $46 \mathrm{XY}$ karyotyping and the SRY mutation gonadal dysgenesis was diagnosed. Because of the risk of malignancy in gonadal dysgenesis and the elevated testosterone level that is not expected in streak gonads), the patient and her parents were counseled by a multidisciplinary team consisting of a pediatric endocrinologist, psychologist and gynecologist, to undergo a prophylactic laparoscopic bilateral gonadectomy because of the risk of malignancy. There was patient delay of one year before the gonadectomy was performed, due to parental problems with accepting the diagnosis. In June 2016, an uncomplicated laparoscopic bilateral gonadectomy was performed. A small sized uterus was seen, and the bilateral gonads were located close to the lateral pelvic wall. The tubes were macroscopically normal and further inspection of the abdomen showed no abnormalities.

Histological examination of the gonads revealed tubes and fimbriae in both gonads, but also epididymis, rete testis and Leydig cells in the hilus. The gonads itself contained mainly ovarian stroma, but also some Leydig cells. There was no normal pre-existent tubule seminiferous and no oocytes. Both gonads also contained a gonadoblastoma and next to that an invasive component conform seminoma/dysgerminoma of 1.0 to $1.5 \mathrm{~cm}$. Two weeks post-operatively we repeated $\mathrm{LDH}$ and testosterone examination; LDH was still elevated $300 \mathrm{U} / \mathrm{l}(\mathrm{ref}<250)$, and the testosterone level was normalized, $0.4 \mathrm{nmol} / \mathrm{l}$. A CT chest and abdomen was performed post-operatively not showing evidence of metastases or lymphadenopathy.

In the Netherlands every oncology patients is discussed in a local multidisciplinary team of gynae-oncologists, a medical oncologist, radiologist, pathologist, radiation oncologist, additionally in our case a pediatric oncologist from the University Medical Centre of Utrecht was consulted, and a strategy of treatment and followup was proposed. Patient and parents gave informed consent. No indication was found for surgical (re-)staging since the CT scan was unremarkable and recurrence of disease could be well treated with chemotherapy. Follow-up for 5 years was proposed starting with frequent visits every three months with tumor markers (inhibine $\mathrm{B}$, alpha foetoprotein, LDH, $\beta \mathrm{HCG}$ ) and an abdominal ultrasound, combined with MRI if the ultrasound is not conclusive. Induction of puberty with hormonal therapy was considered safe. The patient started with $525 \mu \mathrm{g}$ Estradiol 1 capsule a day for the first three months. Moreover, a consultation with a psychologist for the patient and her parents was arranged. During 1,5 year of follow-up there were no signs of recurrence of disease, either clinically, or on imaging and biochemical she is stable. Though, it will take years of follow-up to say our conservative strategy was successful in this rare case of an 18-year-old patient with 46XY, SRY gene mutation and bilateral gonadoblastoma and dysgerminoma.

\section{Discussion}

We describe a rare case of a patient with $46 \mathrm{XY}$ and a SRY gene mutation and bilateral gonadoblastoma and dysgerminoma. Limited data are available about this type of germ cell tumor and the required treatment and follow-up. A conservative approach was chosen in this case, as completion surgery and adjuvant chemotherapy are controversial and recurrence of disease is well treated with chemotherapy. Because of the malignant potential of the $\mathrm{XY}$ digenetic gonads the timing of the (prophylactic) gonadectomy is important to prevent malignancy. In this case the laparoscopic gonadectomy was intended to be prophylactic. However, at the time of surgery, at the age of 18-years, the gonadoblastoma and dysgerminoma had already developed bilaterally. Malignant ovarian germ cell tumors have a peak incidence between the age of $15-19$ years [13]. From that perspective, the surgery should ideally be performed before the age of 15 years. Due to the normal female external genitalia and the fact that the first presentation of the patients normally is with primary amenorrhea, most patients are not diagnosed before the age of 15. In the pre-operative work-up, LDH could be of diagnostic value, as it is known to be a predictive marker for dysgerminoma. In case of gonadoblastoma, estradiol, inhibin B, testosterone and androstenedione can be elevated. These markers can therefore be helpful in the pre-operative diagnosis. Surgery is regarded standard care and laparoscopy is considered safe in a malignant ovarian germ cell tumor $[9,10]$. In advanced stage disease (>=stage 2) or additional risk factors like high differentiation grade, platinum-based combination chemotherapy is advised but this is under debate [9]. Another point of discussion is the need for complete surgical staging. 
In 2016, Park et al. [13] published 2 studies on treatment strategy in young women with FIGO stage 1 malignant ovarian germ cell tumors. One retrospective study of 135 patients, describes the role of complete surgical staging before follow-up with and without adjuvant chemotherapy [13]. The other retrospective study included 31 patients, evaluated the safety of fertility-sparing surgery alone followed by close surveillance [14]. Overlap in patients between these studies is likely since the treatment and follow-up was at the Asian Medical Centre, Seoul, Korea between 1990 and 2014 [13] and between 1992 and 2011 [14]. The 135 patients were identified from an electronic cancer registry database. Eighty-six patients underwent complete surgical staging (62.7\%) and 49 (36.3\%) did not. Complete staging surgery was defined as total hysterectomy, bilateral salphingo-oophorectomy, peritoneal cytology, peritoneal biopsy, omentectomy, or omental biopsy and lymph node evaluation (dissection, sampling, or removal of enlarged lymph nodes). In patients who wished to preserve their fertility, fertility sparing surgery (preservation of the uterus and at least one ovary) was performed as part of the staging. No selection criteria of patients for surgical staging or adjuvant chemotherapy were described. No data about upstaging of disease were available. The recurrence rate of patients who were not staged was higher than that of stage IA-IB ( $p=0.035)$ and stage IC $(p=0.190)$. However, they found that the overall survival outcome was not influenced by complete staging surgery because most of the patients with recurrent disease were successfully salvaged by chemotherapy [13]. In the other study, 31 (17 dysgerminomas, 11 immature teratoma, 1 yolk sac tumor, and 2 mixed malignant ovarian germ cell tumors) patients underwent fertility sparing surgery of whom 20 (65\%) underwent complete surgical staging ( 2 showing positive peritoneal cytology) and 11 patients remained unstated. The recurrence rates were $15 \%(3 / 20)$ and $36.4 \%(4 / 11)$ for completely versus unstated patients $(\mathrm{P}=0.210)$. Four of the patients with dysgerminomas had a recurrence (4/17), 2 of them were unstaged [14]. The 10-year disease-free survival was $77 \%$ and the 10 -year overall survival was $97 \%$, using chemotherapy only in case of disease recurrence. This study showed that by using chemotherapy only in recurrence of disease, unnecessary BEP chemotherapy could be avoided in $77 \%$ of the patients. Park et al. [14] state that to enter a surveillance strategy without adjuvant chemotherapy, complete surgical staging is mandatory [13]. On the other hand, they conclude that fertility sparing surgery alone with surveillance could be a safe treatment strategy, because most recurrences can be successfully salvaged by surgery and BEP chemotherapy [14].

A retrospective study among 123 patients with malignant ovarian germ cell tumors by Mangili et al. [15] described an overall 5-year survival of $97.9 \%$ in patients with stage I disease. In this group of patients, 49 had a dysgerminoma (39.8\%). Mangili et al. [15] state that the radicality of surgery was not an independent predictor of recurrence or death, and conservative surgery did not seem to influence prognosis. Age above 45 years and treatment in a non-referral center were independent risk factors for recurrence, whereas stage greater than I and yolksac histology were independent poor prognostic indicators. With platinum-based multi agent chemotherapy most disease recurrences were successfully salvaged [15]. Therefore, (re-)staging surgery and adjuvant chemotherapy are controversial. Often there are no additional findings, and no upstaging of disease takes place. Furthermore, patients with stage I dysgerminoma, who relapse, seem to be salvaged with secondary cytoreductive surgery and platinum-based chemotherapy. Although this is all based on small numbers of patients. The low rate of recurrence of disease and the high 10-year overall survival using chemotherapy only in recurrences was the reason why we advised not to start with chemotherapy directly after surgery, but to keep BEP chemotherapy as an option if recurrence of disease occurs.

Hormonal replacement therapy may be started after surgery [10]. The Dutch national guideline non non-epithelial malignancies of the ovaries, recommends regular follow-up during 5 years in FIGO stage I tumors with normal post-operative markers after the gonadectomy. By describing our rare case of bilateral dysgerminoma with gonadoblastoma in a 46XY patient and our conservative management we hope to contribute to the general knowledge about the (early) development of dysgerminoma and its management.

\section{Teaching Points}

A. The age of 18 years at time of (prophylactic) surgery is generally late, as malignant ovarian germ cell tumors have a peak incidence between 15-19 years of age. Therefore, prophylactic bilateral gonadectomy is recommended upon diagnosis of XY-gonadal dysgenesis.

B. Changes in tumor markers like LDH, inhibin B, testosterone and androstenedione can be elevated and therefore helpful in the diagnosis of gonadoblastoma and dysgerminoma.

C. There are indications that overall survival outcome of patients with malignant ovarian germ cell tumors is not influenced by complete staging surgery. By using chemotherapy only in recurrence of disease, unnecessary BEP chemotherapy may be avoided in $77 \%$ of patients. This supports the conservative management in the current case. Though, eventually it will take years of follow-up to say our strategy has been successful.

\section{References}

1. Hersmus R, de Leeuw BH, Wolffenbuttel KP, Drop SL, Oosterhuis JW, et al. (2008) New insights into type II germ cell tumor pathogenesis based on studies of patients with various forms of disorders of sex development (DSD). Mol Cell Endocrinol 291(1-2):1-10.

2. Hersmus R, Van der Zwan YG, Stoop H, Bernard P, Sreenivasan R, et al. (2012) A 46, XY female DSD patient with bilateral gonadoblastoma, a novel SRY missense mutation combined with a WT1 KTS splice-site mutation. PloS one 7(7): e40858.

3. Looijenga LH, Hersmus R, De Leeuw BH, Stoop H, Cools M, et al. (2010) Gonadal tumours and DSD. Best Pract Res Clin Endocrinol Metab 24(2): 291-310.

4. Du X, Zhang X, Li Y, Han Y (2014) 46, XY female sex reversal syndrome with bilateral gonadoblastoma and dysgerminoma. Exp Ther Med 8(4): 1102-1104. 
5. Yuce O, Doger E, Celik N, Emeksiz HC, Camurdan MO, et al. (2015) Gonadoblastoma with dysgerminoma in a phenotypically turner-like girl with 45, X/46, XY Karyotype. J Clin Res Pediatr Endocrinol 7(4): 336-339.

6. Coyle D, Kutasy B, Han Suyin K, Antao B, Lynch SA, et al. (2016) Gonadoblastoma in patients with $45, \mathrm{X} / 46, \mathrm{XY}$ mosaicism: A 16-year experience. J Pediatr Urol 12(5): 283.

7. Hermans AJ, Kluivers KB, Janssen LM, Siebers AG, Wijnen MH, et al. (2016) Adnexal masses in children, adolescents and women of reproductive age in the Netherlands: A nationwide population-based cohort study. Gynecologic Oncology 143(1): 93-97.

8. Hanlon AJ, Kimble RM (2015) Incidental gonadal tumors at the time of gonadectomy in women with Swyer syndrome: a case series. Journal of pediatric and adolescent gynecology 28(2): e27-29.

9. Li J, Wu X (2016) Current strategy for the treatment of ovarian germ cell tumors: Role of extensive surgery. Curr Treat Options Oncol 17(8): 44.

10. Zhao T, Liu Y, Jiang H, Zhang H, Lu Y (2016) Management of bilateral malignant ovarian germ cell tumors: Experience of a single institute. Mol Clin Oncol 5(2): 383-387.
11. Cools M, Looijenga LH, Wolffenbuttel KP, Drop SL (2009) Disorders of sex development: update on the genetic background, terminology and risk for the development of germ cell tumors. World J Pediatr 5(2): 93102.

12. Vander Zwan YG, Biermann K, Wolffenbuttel KP, Cools M, Looijenga LH (2015) Gonadal maldevelopment as risk factor for germ cell cancer: towards a clinical decision model. Eur Urol 67(4): 692-701.

13. Park JY, Kim DY, Suh DS, Kim JH, Kim YM, Kim YT, etal. (2016) Significance of the complete surgical staging of stage I malignant ovarian germ cell tumors. Annals of surgical oncology 23(9): 2982-2987.

14. Park JY, Kim DY, Suh DS, Kim JH, Kim YM, et al. (2016) Outcomes of surgery alone and surveillance strategy in young women with stage I malignant ovarian germ cell tumors. Int J Gynecol Cancer 26(5): 859864.

15. Mangili G, Sigismondi C, Gadducci A, Cormio G, Scollo P, et al. (2011) Outcome and risk factors for recurrence in malignant ovarian germ cell tumors: a MITO-9 retrospective study. Int J Gynecol Cancer 21(8): 14141421.
Creative Commons Attribution 4.0 International License

For possible submissions Click Here
Submit Article

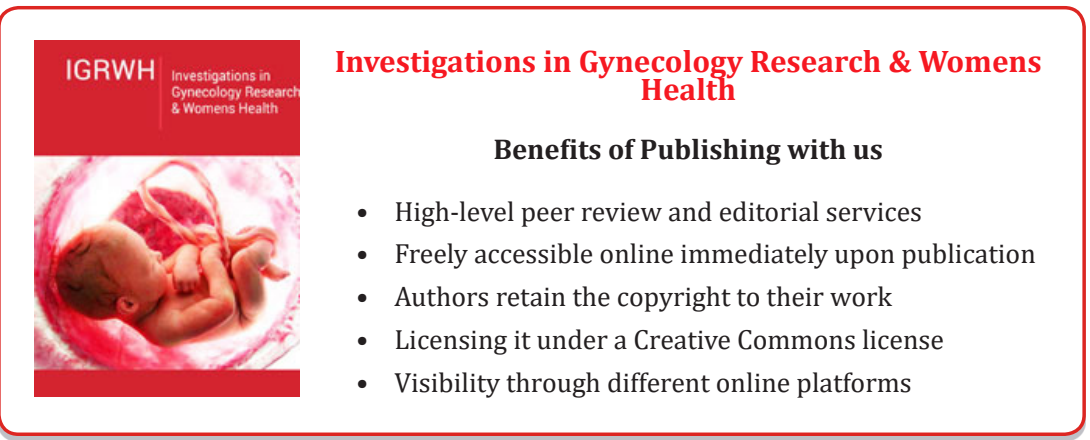

